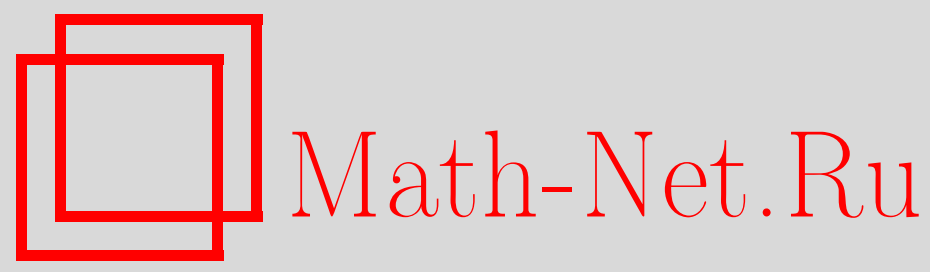

Х. Д. Икрамов, А. М. Назари, Нормальные матрицы и обобщение формулы Малышева, Матем. заметки, 2004, том 75, выпуск 5, 652-662

DOI: https://doi.org/10.4213/mzm61

Использование Общероссийского математического портала Math-Net.Ru подразумевает, что вы прочитали и согласны с пользовательским соглашением http://www . mathnet.ru/rus/agreement

Параметры загрузки:

IP: 34.229 .45 .116

26 апреля 2023 г., $13: 27: 14$ 
УДК 519.6

\title{
НОРМАЛЬНЫЕ МАТРИЦЫ И ОБОБШЕНИЕ ФОРМУЛЫ МАЛЫШЕВА
}

\author{
Х. Д. Икрамов, А. М. Назари
}

Пусть $A$ - комплексная матрица порядка $n, n \geqslant 3$. Сопоставим ей матрицу утроенного порядка

$$
Q(\gamma)=\left(\begin{array}{ccc}
A & \gamma_{1} I_{n} & \gamma_{3} I_{n} \\
0 & A & \gamma_{2} I_{n} \\
0 & 0 & A
\end{array}\right)
$$

где $\gamma_{1}, \gamma_{2}, \gamma_{3}$ - скалярные параметры и $\gamma=\left(\gamma_{1}, \gamma_{2}, \gamma_{3}\right)$. Пусть $\sigma_{i}, 1 \leqslant i \leqslant 3 n,-$ сингулярные числа матрицы $Q(\gamma)$, упорядоченные по убыванию. Доказано, что спектральное расстояние от нормальной матрицы $A$ до множества $\mathscr{M}$ матриц, имеющих собственное значение 0 кратности $\geqslant 3$, равно

$$
\max _{\gamma_{1}, \gamma_{2} \geqslant 0, \gamma_{3} \in \mathbb{C}} \sigma_{3 n-2}(Q(\gamma))
$$

Этот факт является уточнением (в случае нормальных матриц) формулы А. Н. Малышева для спектрального расстояния от произвольной $(n \times n)$-матрицы $A$ до множества $(n \times n)$-матриц, имеющих кратное собственное значение нуль.

Библиография: 2 названия.

1. Введение. Пусть $A$-комплексная $(n \times n)$-матрица, $\mathscr{L}$-множество $(n \times n)$-матриц с кратным собственным значением 0 . А. Н. Мальшевым получена следующая формула для спектрального расстояния от $A$ до $\mathscr{L}$ (см. [1]):

$$
\rho_{2}(A, \mathscr{L})=\min _{L \in \mathscr{L}}\|A-L\|_{2}=\max _{\gamma \geqslant 0} \sigma_{2 n-1}(P(\gamma))
$$

Здесь

$$
P(\gamma)=\left(\begin{array}{cc}
A & \gamma I_{n} \\
0 & A
\end{array}\right)
$$

а $\sigma_{i}(\cdot)$ обозначает $i$-е сингулярное число соответствующей матрицы. В дальнейшем без специального упоминания предполагается, что сингулярные числа всякой матрицы упорядочены по убыванию.

Пусть теперь $\mathscr{M}$ - множество $(n \times n)$-матриц, имеющих собственное значение 0 кратности $\geqslant 3$. Ясно, что $\mathscr{M}$ является подмножеством множества $\mathscr{L}$. Спрашивается, можно ли выразить спектральное расстояние от $A$ до $\mathscr{M}$ точной формулой типа (1)? 
Попытка обосновать такую формулу была предпринята в [2]. Сопоставим матрице $A$ матрицу утроенного порядка

$$
Q(\gamma)=\left(\begin{array}{ccc}
A & \gamma_{1} I_{n} & \gamma_{3} I_{n} \\
0 & A & \gamma_{2} I_{n} \\
0 & 0 & A
\end{array}\right)
$$

где $\gamma_{1}, \gamma_{2}, \gamma_{3}$ - скалярные параметры и $\gamma=\left(\gamma_{1}, \gamma_{2}, \gamma_{3}\right)$. Определим функцию

$$
f(\gamma)=\sigma_{3 n-2}(Q(\gamma)) \text {. }
$$

В [2] показано, что для расстояния $\rho_{2}(A, \mathscr{M})$ справедлива нижняя оценка

$$
\rho_{2}(A, \mathscr{M}) \geqslant \max _{\gamma_{1}, \gamma_{2} \geqslant 0, \gamma \in \mathbb{C}} f(\gamma) .
$$

При этом, если функция (3) имеет локальньй экстремум в какой-либо точке $\gamma^{*}=\left(\gamma_{1}^{*}\right.$, $\left.\gamma_{2}^{*}, \gamma_{3}^{*}\right)$, где

$$
\gamma_{1}^{*}>0, \quad \gamma_{2}^{*}>0,
$$

то (4) превращается в равенство

$$
\rho_{2}(A, \mathscr{M})=\max _{\gamma_{1}, \gamma_{2} \geqslant 0, \gamma_{3} \in \mathbb{C}} f(\gamma) .
$$

В данной статье мы доказываем, что для нормальной матрицы $A$ имеет место именно равенство (6), а не просто неравенство (4). Изложим вкратце соображения, на которых основан этот вывод; они развиваются более подробно в разделах $2-5$.

Пусть

$$
\sigma_{1}(A) \geqslant \cdots \geqslant \sigma_{n}(A)
$$

суть сингулярные числа матрицы $A$. Если

$$
\sigma_{n-2}(A)=\sigma_{n-1}(A)=\sigma_{n}(A),
$$

то, очевидно,

$$
f(0)=\sigma_{n}(A)=\sigma_{n-2}(A) .
$$

В то же время, существует матрица с (по меньшей мере) тройным собственным значением 0 , находящаяся от $A$ на (спектральном) расстоянии $\sigma_{n-2}(A)$, а именно, матрища $B$ с теми же сингулярными векторами, что у $A$, и сингулярными числами $\sigma_{1}(A), \ldots$, $\sigma_{n-3}(A), 0,0,0$. Вместе с (4) и (9), данное наблюдение доказывает равенство (6) для рассматриваемого случая. Заметим, что нормальность матрицы $A$ в этом рассуждении не используется.

Для дальнейшего анализа важны приводимые ниже свойства функции $f$. Они имеют место для произвольной матрицы $A$ и либо обоснованы в [2], либо доказьваются таким же образом, каким в [1] доказьваются свойства функции

$$
g(\gamma)=\sigma_{2 n-1}(P(\gamma)) .
$$


а) Поскольку сингулярные числа матрицы непрерывно зависят от ее элементов, $f$ есть непрерьвная функция от $\gamma_{1}, \gamma_{2}$ и $\gamma_{3}$.

б) Функция $f$ либо тождественно равна нулю (что соответствует ситуации, когда $A \in \mathscr{M})$, либо не обращается в нуль ни в одной точке $\gamma \neq 0$.

в) Имеют место равенства

$$
f\left(-\gamma_{1}, \gamma_{2}, \gamma_{3}\right)=f\left(\gamma_{1}, \gamma_{2},-\gamma_{3}\right), \quad f\left(\gamma_{1},-\gamma_{2}, \gamma_{3}\right)=f\left(\gamma_{1}, \gamma_{2},-\gamma_{3}\right)
$$

Именно они позволяют ограничиться точками ортанта $\gamma_{1} \geqslant 0, \gamma_{2} \geqslant 0$ при вычислении максимумов в правых частях соотношений (4) и (6).

г) Справедливо предельное соотношение

$$
f(\gamma) \rightarrow 0 \quad \text { при } \quad\|\gamma\|_{2}^{2}=\gamma_{1}^{2}+\gamma_{2}^{2}+\left|\gamma_{3}\right|^{2} \rightarrow 0 .
$$

Из перечисленных свойств вытекает: если $A \notin \mathscr{M}$, то существует точка

$$
\gamma^{*}=\left(\gamma_{1}^{*}, \gamma_{2}^{*}, \gamma_{3}^{*}\right)
$$

реализующая глобальньй максимум функции $f$ на множестве

$$
\gamma_{1} \geqslant 0, \quad \gamma_{2} \geqslant 0, \quad \gamma_{3} \in \mathbb{C} \text {. }
$$

Например, в ситуации, описьваемой соотношениями (8), глобальный максимум достигается в точке

$$
\gamma=(0,0,0)
$$

(или, сокращенно, $\gamma=0$ ).

Суть проводимого ниже анализа в том, чтобы показать следующее.

1. Если

$$
\sigma_{n-1}(A)<\sigma_{n-2}(A),
$$

то (12) не может быть точкой максимума функции $f$. Это утверждение обосновьвается в разделах 3 и 4; первый из них относится к случаю, когда

$$
\sigma_{n}(A)<\sigma_{n-1}(A)
$$

а второй - к значительно более сложной ситуации, когда (13) сочетается с равенством

$$
\sigma_{n}(A)=\sigma_{n-1}(A) \text {. }
$$

2. Если $\gamma^{*}$ - точка максимума функции $f$ и

$$
\sigma^{*}=f\left(\gamma^{*}\right)
$$

то $\sigma^{*}$ есть кратное сингулярное число матрицы $Q\left(\gamma^{*}\right)$.

Доказательства утверждений 1 и 2 используют описьваемую в разделе 2 редукцию матрицы $Q(\gamma)$ к прямой сумме $(3 \times 3)$-блоков. Наличие такой редукции существенно упрощает исследование по сравнению со случаем матрищы $A$ общего вида.

Опираясь на утверждения 1 и 2 , мы показьваем в разделе 5 , что в точке $\gamma^{*}$ вьполняются соотношения

$$
\gamma_{1}^{*}=\gamma_{2}^{*}>0 \text {. }
$$

Как было отмечено вьше, эти соотношения гарантируют равенство (6). 
2. Редукция задачи. Пусть $A$ - нормальная $(n \times n)$-матрица со спектральньц разложением

$$
A=W \Lambda W^{*},
$$

где $W$ - унитарная матрица собственных векторов, а

$$
\Lambda=\operatorname{diag}\left(\lambda_{1}, \ldots, \lambda_{n}\right)
$$

Порядок собственных значений в (16) подчинен условиям (7).

Произведем с матрицей (2) унитарное подобие

$$
Q(\gamma) \rightarrow R(\gamma)=U^{*} Q(\gamma) U
$$

где

$$
U=W \oplus W \oplus W
$$

Получим

$$
R(\gamma)=\left(\begin{array}{ccc}
\Lambda & \gamma_{1} I_{n} & \gamma_{3} I_{n} \\
0 & \Lambda & \gamma_{2} I_{n} \\
0 & 0 & \Lambda
\end{array}\right)
$$

Симметричной перестановкой строк и столбцов матрица (17) приводится к прямой сумме

$$
S(\gamma)=\Gamma_{1} \oplus \cdots \oplus \Gamma_{n}
$$

где

$$
\Gamma_{i}=\left(\begin{array}{ccc}
\lambda_{i} & \gamma_{1} & \gamma_{3} \\
0 & \lambda_{i} & \gamma_{2} \\
0 & 0 & \lambda_{i}
\end{array}\right), \quad i=1,2, \ldots, n
$$

Поскольку матрицы $Q(\gamma)$ и $S(\gamma)$ унитарно подобны, справедливо равенство

$$
f(\gamma)=\sigma_{3 n-2}(S(\gamma))
$$

В силу разложения (18) сингулярный спектр матрицы $S(\gamma)$ есть объединение сингулярных спектров блоков $\Gamma_{1}, \ldots, \Gamma_{n}$. Это обстоятельство широко используется в дальнейшем.

3. Сингулярные числа матрицы Г. Для последующего анализа нам необходимы несколько простых оценок, связанных с сингулярными числами матрицы

$$
\Gamma(\gamma)=\left(\begin{array}{ccc}
a & \gamma_{1} & \gamma_{3} \\
0 & a & \gamma_{2} \\
0 & 0 & a
\end{array}\right)
$$

где $a-$ произвольное комплексное число.

ЛЕмма 1. Если $\sigma_{1}(\gamma)$ - старшее сингулярное число матрицы (19), то

$$
\sigma_{1}(\gamma) \geqslant \max \left\{\left(|a|^{2}+\left|\gamma_{1}\right|^{2}+\left|\gamma_{3}\right|^{2}\right)^{1 / 2},\left(|a|^{2}+\left|\gamma_{2}\right|^{2}+\left|\gamma_{3}\right|^{2}\right)^{1 / 2}\right\} .
$$


ДокАЗАТЕЛЬСТвО. Числа в правой части оценки (20) суть евклидовы длины первой строки и третьего столбца матрицы $\Gamma(\gamma)$. Сама эта оценка есть простое следствие хорошо известных экстремальных описаний числа $\sigma_{1}$ :

$$
\sigma_{1}=\max _{x \neq 0} \frac{\|\Gamma x\|_{2}}{\|x\|_{2}}=\max _{x \neq 0} \frac{\left\|\Gamma^{*} x\right\|_{2}}{\|x\|_{2}}
$$

СЛЕДСТВИЕ 1. Если Хотя бъ одно из чисел $\gamma_{1}, \gamma_{2}, \gamma_{3}$ отлично от нуля, то

$$
\sigma_{1}(\gamma)>\sigma_{1}(0)=|a|
$$

ЛЕмма 2. Пусть $\sigma_{3}(\gamma)$ - младиее сингулярное число матрицы (19). Если ағ0 и хотя бы одно из чисел $\gamma_{1}, \gamma_{2}, \gamma_{3}$ отлично от нуля, то

$$
\sigma_{3}(\gamma)<\sigma_{3}(0)=|a|
$$

ДокАЗАТЕЛЬСтво. Применим к матрище

$$
\Gamma^{-1}(\gamma)=\frac{1}{a^{3}}\left(\begin{array}{ccc}
a^{2} & -a \gamma_{1} & \gamma_{1} \gamma_{2}-a \gamma_{3} \\
0 & a^{2} & -a \gamma_{2} \\
0 & 0 & a^{2}
\end{array}\right)
$$

оценку, аналогичную неравенству (20). Из нее следует

$$
\sigma_{1}\left(\Gamma^{-1}(\gamma)\right)=\frac{1}{\sigma_{3}(\gamma)} \geqslant \frac{1}{|a|^{2}} \max \left\{\sqrt{|a|^{2}+\left|\gamma_{1}\right|^{2}}, \sqrt{|a|^{2}+\left|\gamma_{2}\right|^{2}}\right\}
$$

откуда

$$
\sigma_{3}(\gamma) \leqslant \min \left\{\frac{|a|^{2}}{\sqrt{|a|^{2}+\left|\gamma_{1}\right|^{2}}}, \frac{|a|^{2}}{\sqrt{|a|^{2}+\left|\gamma_{2}\right|^{2}}}\right\} .
$$

Это неравенство дает требуемое соотношение $(22)$, если хотя бы одно из чисел $\gamma_{1}$ и $\gamma_{2}$ отлично от нуля. В противном случае, $\gamma_{3} \neq 0$ и из той же оценки типа $(20)$ вьводим

$$
\sigma_{3}(\gamma) \leqslant \frac{|a|^{2}}{\sqrt{|a|^{2}+\left|\gamma_{3}\right|^{2}}}
$$

что снова приводит к (22).

Доказанные леммы позволяют дать простое обоснование утверждений 1 и 2, сформулированных во введении статьи, в случае $\sigma_{n}(A)<\sigma_{n-1}(A)$. Действительно, в этом случае в некоторой окрестности точки $\gamma=0$ тремя младшими сингулярными числами матриц $Q(\gamma)$ и $S(\gamma)$ являются сингулярные числа блока

$$
\Gamma_{n}=\left(\begin{array}{ccc}
\lambda_{n} & \gamma_{1} & \gamma_{3} \\
0 & \lambda_{n} & \gamma_{2} \\
0 & 0 & \lambda_{n}
\end{array}\right)
$$


Согласно $(21)$ для точек $\gamma \neq 0$ в этой окрестности имеем

$$
f(\gamma)=\sigma_{1}\left(\Gamma_{n}\right)>\sigma_{1}(0)=\left|\lambda_{n}\right|=f(0)
$$

Таким образом, в точке $\gamma=0$ нет даже локального максимума функции $f(\gamma)$.

Фиксируем неотрицательные числа $\alpha$ и $\beta$ и комплексное число $\mu$, не равные нулю одновременно. Рассмотрим динамику сингулярных чисел матрицы $S(\gamma)$ при движении от точки $\gamma=0$ вдоль луча

$$
\gamma_{1}=\alpha t, \quad \gamma_{2}=\beta t, \quad \gamma_{3}=\mu t
$$

Согласно $(20)$ число $\sigma_{1}\left(\Gamma_{n}\right)$ неограниченно возрастает с ростом $t$. Поэтому максимум функции $f$ вдоль луча (26) достигается при значении $t=t_{0}$, соответствующем точке пересечения графика $\sigma_{1}\left(\Gamma_{n}\right)$ с графиком младшего сингулярного числа одного из блоков $\Gamma_{i}, i=1, \ldots, n-1$. Поскольку точка $\gamma^{*}$ глобального максимума является одновременно максимумом вдоль соответствующего луча, отсюда следует утверждение 2 . Отметим, что сингулярные числа, значения которых совпадают в точке максимума, соответствуют собственным значениям $\lambda_{i}$ и $\lambda_{n}$ различного модуля. Это наблюдениебудет использовано в разделе 5 .

4. Случай $\sigma_{n}(A)=\sigma_{n-1}(A)$. Этот случай значительно сложнеепредыдущего. Мы исследуем его вначале при дополнительном предположении: два собственных значения матрицы $A$, имеющие наименьший модуль, удовлетворяют условию

$$
\lambda_{n-1}+\lambda_{n} \neq 0
$$

Это означает, в частности, что $\lambda_{n} \neq 0$.

Покажем, что при условии (27) существуют луч $(26)$ и некоторая окрестность $\mathscr{O}$ точки $t=0$ на этом луче такие, что $\sigma_{2}\left(\Gamma_{n}\right)$ и $\sigma_{2}\left(\Gamma_{n-1}\right)$ суть монотонно возрастающие функции от $t$ в пределах $\mathscr{O}$. Отсюда будет вытекать утверждение 1 . Действительно, для ненулевых $t \in \mathscr{O}$ имеем

$$
f(\gamma)=\min \left\{\sigma_{2}\left(\Gamma_{n}\right), \sigma_{2}\left(\Gamma_{n-1}\right)\right\}>\sigma_{2}(0)=\sigma_{n-1}(A)=f(0)
$$

Нам будет удобнее вместо самих чисел $\sigma_{i}\left(\Gamma_{n}\right)$ рассматривать их квадраты, являющиеся собственньми значениями эрмитовой матрицы

$$
G_{n}=\Gamma_{n}^{*} \Gamma_{n}=|a|^{2} I_{3}+t\left(\bar{a} M+a M^{*}\right)+t^{2} M^{*} M
$$

Здесь

$$
a=\lambda_{n}, \quad M=\left(\begin{array}{ccc}
0 & \alpha & \mu \\
0 & 0 & \beta \\
0 & 0 & 0
\end{array}\right) .
$$

Аналогичная интерпретация при необхдимости применяется к сингулярным числам других блоков $\Gamma_{i}$.

Для малых $t$ справедливы представления

$$
\sigma_{i}^{2}\left(\Gamma_{n}\right)=|a|^{2}+\delta_{i} t+\mathscr{O}\left(t^{2}\right), \quad i=1,2,3
$$


где коэффициенты при линейных членах $\delta_{1}, \delta_{2}, \delta_{3}$ суть собственные значения эрмитовой матрицы

$$
H=\bar{a} M+a M^{*}=\left(\begin{array}{ccc}
0 & \alpha \bar{a} & \mu \bar{a} \\
\alpha a & 0 & \beta \bar{a} \\
\bar{\mu} a & \beta a & 0
\end{array}\right)
$$

Фиксируем положительные числа $\alpha, \beta$ и выберем число $\mu=x+i y$ так, чтобы $\operatorname{det} H$ был отрицательным числом. Поскольку

$$
\operatorname{det} H=\alpha \beta|a|^{2}(\mu a+\bar{\mu} \bar{a})
$$

это равносильно тому, что

$$
\operatorname{Re}(\mu a)=\left(\operatorname{Re} \lambda_{n}\right) x-\left(\operatorname{Im} \lambda_{n}\right) y<0 .
$$

Нулевой след матрицы $H$ и отрицательность ее определителя вместе означают, что два из чисел $\delta_{1}, \delta_{2}, \delta_{3}$ положительны, а третье отрицательно. Отсюда следует, что при движении из нуля вдоль луча (26) два сингулярных числа клетки $\Gamma_{n}$ возрастают, а третье убьвает.

Наложим на выбор числа $\mu$ еще одно ограничение, а именно

$$
\operatorname{Re}\left(\mu \lambda_{n-1}\right)=\left(\operatorname{Re} \lambda_{n-1}\right) x-\left(\operatorname{Im} \lambda_{n-1}\right) y<0 .
$$

Условие (27) обеспечивает, что система неравенств (28), (29) имеет решение $\mu=x+i y$. Для соответствующего луча (26) сингулярные числа клетки $\Gamma_{n-1}$ ведут себя так же, как для $\Gamma_{n}$, т.е. два из них возрастают, а третье убьвает. В частности, $\sigma_{2}\left(\Gamma_{n-1}\right)$, как и $\sigma_{2}\left(\Gamma_{n}\right)$, растет с ростом $t$. Тем самым, утверждение 1 установлено.

Предыдущие рассуждения относились к значениям $t$, принадлежащим окрестности $\mathscr{O}$. Покажем, что и при дальнейшем продвижении вдоль луча $(26)$ рост $\sigma_{2}\left(\Gamma_{n}\right)$ и $\sigma_{2}\left(\Gamma_{n-1}\right)$ продолжится по крайней мере до тех пор, пока графики этих чисел не пересекутся с графиком какого-либо из чисел $\sigma_{3}\left(\Gamma_{i}\right), i=1, \ldots, n-2$.

Предположим противное; пусть, например, $\sigma_{2}\left(\Gamma_{n}\right)$ имеет локальньй максимум $\sigma_{2}^{*}$ при $t=t_{0}$, причем $\sigma_{2}^{*}$ не является сингулярным числом ни для одного из блоков $\Gamma_{i}$, $i=1, \ldots, n-2$.

Повторяя для матрицы $\Gamma_{n}$ те же рассуждения, какие в [2] были применены к блочной матрице $Q(\gamma)$, приходим к следующему заключению: существуют относяшиеся к $\sigma_{2}^{*}$ нормированные правый сингулярньй вектор

$$
u=\left(\begin{array}{l}
u_{1} \\
u_{2} \\
u_{3}
\end{array}\right)
$$

и соответствующий левый сингулярньй вектор

$$
v=\frac{1}{\sigma_{2}^{*}} \Gamma_{n} u=\left(\begin{array}{l}
v_{1} \\
v_{2} \\
v_{3}
\end{array}\right)
$$


такие, что

$$
\begin{array}{lll}
\left|v_{1}\right|^{2}=\left|u_{1}\right|^{2}, & \left|v_{2}\right|^{2}=\left|u_{2}\right|^{2}, & \left|v_{3}\right|^{2}=\left|u_{3}\right|^{2} \\
\bar{v}_{1} v_{2}=\bar{u}_{1} u_{2}, & \bar{v}_{1} v_{3}=\bar{u}_{1} u_{3}, & \bar{v}_{2} v_{3}=\bar{u}_{2} u_{3} .
\end{array}
$$

(Эти равенства суть скалярньй аналог матричного соотношения $V^{*} V=U^{*} U$, полученного в следствии из леммы 6 в [2].)

Из соотношений (32) вытекает, что

$$
v=e^{i \varphi} u, \quad \varphi \in \mathbb{R} .
$$

Подставляя (33) в (31), имеем

$$
\Gamma_{n} u=e^{i \varphi} \sigma_{2}^{*} u
$$

т.е. $u$ должен быть собственным вектором матрищы $\Gamma_{n}$ для собственного значения $e^{i \varphi} \sigma_{2}^{*}$. Однако такого собственного значения у $\Gamma_{n}$ нет: все три ее собственных значения равны $\lambda_{n}$, тогда как

$$
\left|e^{i \varphi} \sigma_{2}^{*}\right|=\sigma_{2}^{*}>\left|\lambda_{n}\right|
$$

Те же соображения применимы и к блоку $\Gamma_{n-1}$. Поэтому функция

$$
f(\gamma)=\min \left\{\sigma_{2}\left(\Gamma_{n}\right), \sigma_{2}\left(\Gamma_{n-1}\right\}\right.
$$

возрастает вдоль луча (26), пока при некотором $t=t_{0}$ график $f(\gamma)$ не пересечется с графиком некоторого числа $\sigma_{3}\left(\Gamma_{i}\right), i=1, \ldots, n-2$.

Отметим, что в проведенном вьше рассуждении локальньй максимум функции $\sigma_{2}\left(\Gamma_{n}\right)$ может быть заменен ее локальным минимумом (лемма 6 в [2] справедлива для любого локального экстремума). Поэтому, если вдоль какого-то луча (26) $\sigma_{2}\left(\Gamma_{n}\right)$ убьвает при $t=0$, то это убьвание сохранится и в дальнейшем. На лучах этого типа, следовательно, глобальньй максимум функции $f$ достигаться не может. Он достигается на некотором луче рассмотренного ранее типа и, как вытекает из нашего анализа, $\sigma^{*}=f\left(\gamma^{*}\right)$ есть кратное сингулярное число матрицы $Q\left(\gamma^{*}\right)$. При этом сингулярные числа, значения которых совпадают в точке $\gamma^{*}$, соответствуют собственным значениям $\lambda_{n}$ и $\lambda_{i}$ (или $\lambda_{n-1}$ и $\lambda_{i}$ ), $i \in\{1, \ldots, n-2\}$, различного модуля.

Нам остается рассмотреть случай

$$
\lambda_{n-1}=-\lambda_{n}
$$

В этом случае движение из нуля вдоль любого луча (26) с ненулевым $\mu$ не приводит к увеличению функции $f$ по сравнению с

$$
f(0)=\sigma_{n}(A)=\sigma_{n-1}(A)
$$

Действительно, одно из неравенств (28) и (29) будет нарушено и соответствуюшее сингулярное число $\sigma_{2}$ будет убьвать вдоль луча. Оно и является значением функции $f$.

Покажем, что, напротив, при движении по лучу

$$
\gamma_{1}=\alpha t, \quad \gamma_{2}=\beta t, \quad \gamma_{3}=0, \quad \alpha, \beta>0,
$$


число $\sigma_{2}\left(\Gamma_{n}\right)$ всегда будет больше числа $(35)$, если $t>0$. Это же верно для $\sigma_{2}\left(\Gamma_{n-1}\right)$. В действительности, вдоль луча (36) блоки $\Gamma_{n-1}$ и $\Gamma_{n}$ имеют одни и те же сингулярные числа, поскольку $G_{n-1}=\Gamma_{n-1}^{*} \Gamma_{n-1}$ и $G_{n}=\Gamma_{n}^{*} \Gamma_{n}$ (с точностью до диагонального подобия) совпадают с одной и той же матрицей

$$
G=|a|^{2} I_{3}+\left(\begin{array}{ccc}
0 & \alpha \bar{a} t & 0 \\
\alpha a t & \alpha^{2} t^{2} & \beta \bar{a} t \\
0 & \beta \alpha t & \beta^{2} t^{2}
\end{array}\right)=|a|^{2} I_{3}+H .
$$

Здесь $|a|=\left|\lambda_{n-1}\right|=\left|\lambda_{n}\right|=\sigma_{n}(A)$.

Присутствие в матрище $H$ положительно полуопределенного $(2 \times 2)$-блока

$$
\left(\begin{array}{cc}
0 & 0 \\
0 & \beta^{2} t^{2}
\end{array}\right)
$$

свидетельствует, что ее второе собственное значение $\lambda_{2}(H)$ неотрицательно. На самом деле,

$$
\lambda_{2}(H)>0
$$

так Kak

$$
\operatorname{det} H=-\alpha^{2} \beta^{2}|a|^{2} t^{4} \neq 0
$$

при $t \neq 0$. Неравенство (37) означает, что

$$
\sigma_{2}\left(\Gamma_{n}\right)>|a|=f(0), \quad \text { если } t>0 .
$$

Итак, утверждение 1 справедливо и в этом случае. Утверждение 2 обосновьвается так же, как и вьшее.

5. Глобальный максимум функции $f(\gamma)$. В двух предыдущих разделах установлено: глобальньй максимум функции $f(\gamma)$ может достигаться лишь в точках $\gamma$, где одно из сингулярыых чисел блока $\Gamma_{n}$ (или блока $\Gamma_{n-1}$; см. раздел 4 ) совпадает с младшим сингулярньм числом одного из блоков $\Gamma_{i}$. При этом $i \in\{1,2, \ldots, n-1\}$ в разделе 3 и $i \in\{1,2, \ldots, n-2\}$ в разделе 4 . Во всех случаях совпадающие сингулярные числа относятся к блокам $\Gamma$, порожденным собственными значениями различного модуля. Пусть $a(b)$ то из них, которое имеет больший (меньший) модуль. Соответствуюшие им блоки обозначим через $\Gamma_{a}$ и $\Gamma_{b}$.

Характеристический многочлен матрищы $G_{a}=\Gamma_{a}^{*} \Gamma_{a}$ имеет вид

$$
\lambda^{3}+\alpha_{1} \lambda^{2}+\alpha_{2} \lambda+\alpha_{3}=0
$$

где

$$
\begin{aligned}
& \alpha_{1}=-\operatorname{tr} G_{a}=-\left(3|a|^{2}+\gamma_{1}^{2}+\gamma_{2}^{2}+\left|\gamma_{3}\right|^{2}\right), \\
& \alpha_{2}=3|a|^{4}+\left(\gamma_{1}^{2}+\gamma_{2}^{2}+\left|\gamma_{3}\right|^{2}\right)|a|^{2}+\gamma_{1}^{2} \gamma_{2}^{2}-2 \gamma_{1} \gamma_{2} \operatorname{Re}\left(\gamma_{3} a\right) \\
& \alpha_{3}=-\left|\operatorname{det} \Gamma_{a}\right|^{2}=-|a|^{6}
\end{aligned}
$$

Аналогичные выражения справедливы для коэффициентов характеристического многочлена

$$
\lambda^{3}+\beta_{1} \lambda^{2}+\beta_{2} \lambda+\beta_{3}=0
$$


матрицы $G_{b}=\Gamma_{b}^{*} \Gamma_{b}$.

По предположению матрицы $G_{a}$ и $G_{b}$ имеют общее собственное значение, которое удовлетворяет обоим уравнениям (38) и (39), а потому и квадратному уравнению

$$
\left(\alpha_{1}-\beta_{1}\right) \lambda^{2}+\left(\alpha_{2}-\beta_{2}\right) \lambda+\alpha_{3}-\beta_{3}=0
$$

или приведенному уравнению

$$
\lambda^{2}+r(\gamma) \lambda+s(\gamma)=0
$$

Здесь

$$
r(\gamma)=-\left(|a|^{2}+|b|^{2}\right)-\frac{1}{3}\left(\gamma_{1}^{2}+\gamma_{2}^{2}+\left|\gamma_{3}\right|^{2}\right)+\frac{2}{3} \gamma_{1} \gamma_{2} \frac{\operatorname{Re}\left[\gamma_{3}(a-b)\right]}{|a|^{2}-|b|^{2}}
$$

a $s(\gamma)$, в действительности, не зависит от $\gamma$ :

$$
s(\gamma)=\frac{1}{3}\left(|a|^{4}+|b|^{4}+|a|^{2}|b|^{2}\right) .
$$

Положим $\gamma_{3}=\xi_{3}+i \xi_{4}$. Тогда коэффициент $r(\gamma)$ есть гладкая функция от вешественных переменных $\gamma_{1}, \gamma_{2}, \xi_{3}, \xi_{4}$. Поэтому корни уравнения (40) там, где они различны, тоже гладко зависят от $\gamma_{1}, \gamma_{2}, \xi_{3}, \xi_{4}$.

Точке глобального максимума $\gamma^{*}$ соответствует экстремум по $\gamma$ корня $\lambda(\gamma)$ уравнения (40). Дифференцируя равенство (40), нетрудно убедиться, что необходимые условия экстремума

$$
\frac{\partial \lambda}{\partial \gamma_{1}}=\frac{\partial \lambda}{\partial \gamma_{2}}=\frac{\partial \lambda}{\partial \xi_{3}}=\frac{\partial \lambda}{\partial \xi_{4}}=0
$$

равносильны условиям

$$
\frac{\partial r}{\partial \gamma_{1}}=\frac{\partial r}{\partial \gamma_{2}}=\frac{\partial r}{\partial \xi_{3}}=\frac{\partial r}{\partial \xi_{4}}=0 .
$$

Первые два условия (41) имеют вид

$$
-\frac{2}{3} \gamma_{1}+\frac{2}{3} \gamma_{2} \frac{\operatorname{Re}\left[\gamma_{3}(a-b)\right]}{|a|^{2}-|b|^{2}}=0, \quad-\frac{2}{3} \gamma_{2}+\frac{2}{3} \gamma_{1} \frac{\operatorname{Re}\left[\gamma_{3}(a-b)\right]}{|a|^{2}-|b|^{2}}=0
$$

С учетом того, что $\gamma_{1}$ и $\gamma_{2}$ должны быть неотрищательными числами, имеем

$$
\gamma_{1}=\gamma_{2}
$$

Для оставшихся условий (41) положим

$$
a-b=c+i d, \quad c, d \in \mathbb{R} \text {. }
$$

Тогда $\operatorname{Re}\left[\gamma_{3}(a-b)\right]=c \xi_{3}-d \xi_{4}$ и

$$
\begin{aligned}
& \frac{\partial r}{\partial \xi_{3}}=-\frac{2}{3} \xi_{3}+\frac{2}{3} \gamma_{1} \gamma_{2} \frac{c}{|a|^{2}-|b|^{2}} \\
& \frac{\partial r}{\partial \xi_{4}}=-\frac{2}{3} \xi_{4}-\frac{2}{3} \gamma_{1} \gamma_{2} \frac{d}{|a|^{2}-|b|^{2}}
\end{aligned}
$$


Если предположить, что в точке максимума $\gamma^{*}$ координаты $\gamma_{1}$ и $\gamma_{2}$ обращаются в нуль, то условия

$$
\frac{\partial r}{\partial \xi_{3}}=\frac{\partial r}{\partial \xi_{4}}=0
$$

приводят к равенствам $\xi_{3}^{*}=\xi_{4}^{*}=0$. Это означало бы, что $\gamma^{*}=0$ в противоречие с утверждением 1.

Итак, должно быть

$$
\gamma_{1}^{*}=\gamma_{2}^{*}>0
$$

что влечет за собой справедливость формулы (6).

\section{СПИСОК ЦИТИРОВАННОЙ ЛИТЕРАТУРЫ}

[1] Malyshev A. N. A formula for the 2-norm distance from a matrix to the set of matrices with multiple eigenvalues // Numer. Math. 1999. V. 83. P. 443-454.

[2] Икрамов Х. Д., Назари А. М. О расстоянии до ближайшей матрицы с тройным собственным значением нуль // Матем. заметки. 2003. Т. 73. № 4. С. 545-555.

Московский государственный университет им. М.В. Ломоносова 